\title{
EDITORIAL
}

\section{Celebrating the 80th anniversary of electroconvulsive therapy}

\author{
Salih Selek, ${ }^{1}$ (iD) João Quevedo ${ }^{2,3,4,5}$ (iD \\ ${ }^{1}$ Treatment-Resistant Mood Disorders Program, Department of Psychiatry and Behavioral Sciences, McGovern Medical School, The University \\ of Texas Health Science Center at Houston (UTHealth), Houston, TX, USA. ${ }^{2}$ Translational Psychiatry Program, Department of Psychiatry and \\ Behavioral Sciences, McGovern Medical School, UTHealth, Houston, TX, USA. ${ }^{3}$ Center of Excellence on Mood Disorders, Department of \\ Psychiatry and Behavioral Sciences, McGovern Medical School, UTHealth, Houston, TX, USA. ${ }^{4}$ Neuroscience Graduate Program, The \\ University of Texas MD Anderson Cancer Center UTHealth Graduate School of Biomedical Sciences, Houston, TX, USA. ${ }^{5}$ Laboratório de \\ Psiquiatria Translacional, Programa de Pós-Graduação em Ciências da Saúde, Universidade do Extremo Sul Catarinense (UNESC), Criciúma, \\ SC, Brazil. iD SS https://orcid.org/0000-0001-5197-5682, iD JQ https://orcid.org/0000-0003-3114-6611
}

April 2018 had a special day in the history of the modern psychiatry: the 80th anniversary of the first electroconvulsive therapy (ECT) treatment, performed by Ugo Cerletti on a reportedly catatonic patient brought from a railway station in Milan. After 11 courses of treatment, the patient was discharged in July 1938 with full recovery - one of the rarest accomplishments ever seen in psychiatry at the time. ${ }^{1}$ Inducing shock-like states or seizures had long been known to be effective in some mental illnesses, prompting the development of chemical convulsive therapy and insulin therapy. However, both had significant complications, which drove researchers to look for a safer method that had yet to be invented. Wagner-Jauregg's method of causing high fevers by inoculating neurosyphilis patients with malaria cured their psychiatric symptoms in about half of cases, but resulted in many deaths; nevertheless, it earned him the Nobel Prize in $1927 .^{2}$ In this context, ECT was a breakthrough and milestone, with side effects comparable to those of other physical treatments while needing less staffing and observation. However, the vision of a patient "suffering" seizures and other complications (muscle relaxants were not used at the time) was a stain on the image of ECT, and forms the basis of the stigma which persists to this day. ${ }^{3}$

Actually, Dr. Cerletti's dream was to place ECT in a bottle. Per his theory, a humoral substance he called "acro-agonine" was released during epileptic discharges and treated mood disorders; he went so far as to inject shocked-brain emulsions in an attempt to cure patients. However, despite many advances in neuroscience since then, the supposedly curative molecule "released" during ECT has yet to be found. Instead, a growing literature suggests that ECT helps "regrow" neurons in the hippocampus, with particular emphasis on its induction of neuroplasticity and restorative effect on neurocircuitry, therefore making longstanding concerns about brain damage very unlikely.

Correspondence: Salih Selek

E-mail: Salih.selek@uth.tmc.edu
ECT has evolved over the years. The routine administration of neuromuscular blockers and anesthesia somewhat softened its increasingly unpleasant image, and novel methods such as brief-pulse and ultra-brief pulse squarewave stimuli provided better tolerability. Despite new advances in neuromodulation, such as TMS, VNS (both FDA-approved), and other trials, most international guidelines still suggest ECT as an indispensable option for treatment-resistant mood disorders and other selected indications. ${ }^{4}$

Despite its well-established efficacy, ECT is still underutilized. Only approximately $1 \%$ of patients are expected to have this treatment even indicated. A striking statistic is that, in 2017, only 2,773 patients received ECT in the state of Texas (the second largest U.S. state, population about 29 million). Educating the new generation of physicians and psychiatrists with high-quality information on ECT and standing up for this treatment is therefore crucial to prevent orphaning.

\section{Acknowledgements}

The Translational Psychiatry Program (USA) is funded by the Department of Psychiatry and Behavioral Sciences, McGovern Medical School, The University of Texas Health Science Center at Houston (UTHealth). The Center of Excellence on Mood Disorders (USA) is funded by the Pat Rutherford Jr. Chair in Psychiatry, John S. Dunn Foundation, and Anne and Don Fizer Foundation Endowment for Depression Research. The Laboratório de Psiquiatria Translacional (Brazil) is funded by grants from Conselho Nacional de Desenvolvimento Científico e Tecnológico (CNPq), Coordenação de Aperfeiçoamento de Pessoal de Nível Superior (CAPES), Fundação de Amparo à Pesquisa e Inovação do Estado de Santa Catarina (FAPESC), Instituto Cérebro e Mente, and Universidade do Extremo Sul Catarinense (UNESC). JQ is a $1 \mathrm{~A} \mathrm{CNPq}$ Research Fellow.

How to cite this article: Selek S, Quevedo J. Celebrating the 80th anniversary of electroconvulsive therapy. Braz J Psychiatry. 2019;41:7-8. http://dx.doi.org/10.1590/1516-4446-2018-4102 


\section{Disclosure}

$J Q$ has received clinical research support from Allergan and Janssen Pharmaceuticals; has served as speaker for Assurex Health, Daiichi Sankyo, and Janssen Pharmaceuticals; is a stockholder at Instituto de Neurociencias Dr. Joao Quevedo; and receives royalties from Artmed Editora, Artmed Panamericana, and Elsevier. The other author reports no conflicts of interest.

\section{References}

1 Shorter E, Healy D. Shock therapy: a history of electroconvulsive treatment in mental illness. New Brunswick: Rutgers University Press; 2007.

2 Vogel G. Malaria as lifesaving therapy. Science. 2013;342:686.

3 Rzesnitzek L, Lang S. 'Electroshock therapy' in the Third Reich. Med Hist. 2017;61:66-88.

4 Carvalho AF, Vieta E, editors. The treatment of bipolar disorder: integrative clinical strategies and future directions. Oxford: Oxford University Press; 2017. 